\title{
RF tests of a band overlap free DAW accelerating structture
}

\author{
R. Parodi, A.Stella \\ INFN GENOVA \\ Via Dodecaneso 33 \\ 16146 GENOVA ITALY \\ P.Fernandes \\ IMA-CNR \\ Via Leon Battista Alberti \\ Genova Italy
}

\section{Abstract}

Our paper deal with the results of measurements on a six cells Disk-and-Washer structure.

The RF structure operating at $3600 \mathrm{Mhz}$ is optimized for a $\mathrm{BETA}=1$ electron beam, trying to avoid the overlap between the band of the accelerating modes and the bands of the dangerous beam deflecting modes.

The cavity geometry was not optimized for the maximum shunt impdance, but nevertheless a characteristic shunt impedance Z/Q of 1500 ohm was obtained.

Measurements on the six cells prototype section built following our design shown a perfect agreement (in frequency and field istribution) with our computation.

The measured characteristic shunt impedance $Z / Q$ of our prototype was 1500 ohm in agreement with our computations.

The dangerous dipole modes inducing beam breakup were(ascomputed) $40 \mathrm{MHz}$ apart from the operating frequency of the structure showing the correctnessof our assumption.

\section{INTRODUCTION}

The possibility of using a Disk and Washer structure for an High intensity High gain R.F. linac was studied in many laboratories $[1,2,3]$ due to the benefit of high shunt impedance and large bandwidth of the accelerating mode.

Nevertheless the structure is not widely used for practical applications due to the major drawback of the overlap of the accelerating and coupling TM like monopolar bands with the Hybrid dipolar bands.

This band overlap could induce serious beam unstabilities leading to beam break-up at a current lower than the design current of the accelerator.

Due to our interest for a compact, high gradient and efficient Standing Wawe linac structure to be used to build a low energy test accelerator for high energy detectors, we started a research programme on the DAW structure.

Our goal was to stale whether the band overlap is a general feature of the DAW linac or that drawback can be overcome by a suitable choice of the parameters of the clementary cells of the linac structure.
We focused our attention mainly on the mode pattern of the resonant structure without attempting any optimization of the shunt impedance and efficiency of the structure itself.

We succeeded to design a DAW structure free from band overlap in the accelerating mode region, and with a reasonable shunt impedance ( $\mathrm{Z} / \mathrm{Q}$ ) value of $1550 \mathrm{ohm}$ at a frequency of $3650 \mathrm{Mhz}$

\section{METHOD OF DESIGN}

For the design of the structure we widely used computer codes for the computation of RF fields in SWresonant structures.

We computed the first ten TE and TM monopolar modes of a linac unit cell by our OSCAR2D [ 4 ]code and the multipolar modes by the URMELT codel 5 ] (courtesy of T. Weiland DESY).

In that way , by using a single cell of the linac and changing the boundary condition on suitable segments of the cell, we where obtain the values of the resonant frequency of the ZERO, PI and PI over TWO modes of the band pass under examination.

From those values we easily reconstructed the dispersion relation of that band pass of the structure [ 6 ]and checked whether for that geometry we have band overlap or not.

Once the right cavity shape had been found, the geometry was slightly changed till the perfect coalescence on the PI mode of the coupling mode bandpass and accelerating mode bandpass was obtained closing the stop band between the two PI modes.

After that a new complete search for the monopolar and multipolar modes was done to eventually detect a hand overlap introduced by the previous geometry changes

The process converged after three iterations leading to the coalescent, band overlap free cell shown in figure 1 .

From our simulations it comes thath the frequency of the accelerating and coupling monopolar TM modes is mainly related to the diameter of the outer region in the washer

zone. 
The frequency of the dipolar hybrid modes is more sensitive to changes in the radial dimension of the disk rergion.

Having observed this property of the unit cell of our structure, we easilly succeeded to design to design a band overlap free DAW.

Further we computed also the frequency distribution for the quadrupolar modes to be sure that also this modes does not affect the frequency spectrum of the accelerating structure inducing potentially harmful beam-break-up modes.

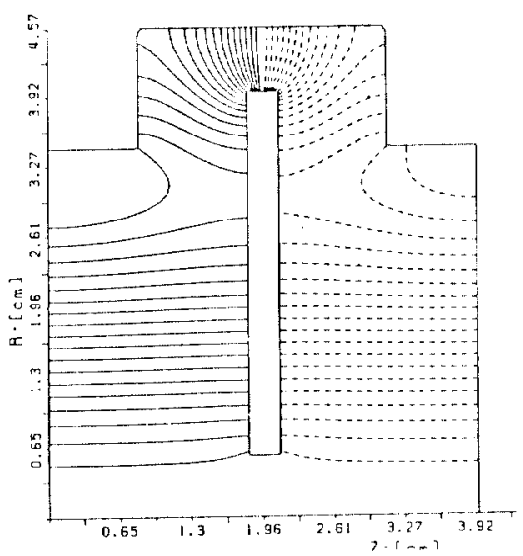

Figure 1. Prototype cell

The relevant RF properties of the cavity are reported in TABLE I.

TABLE I

\section{GEOMETRY AND CAVITY PARAMETERS}

$$
\begin{array}{cccc}
\mathrm{f}[\mathrm{MHz}] & 3668 . & \mathrm{R} \quad[\mathrm{cm}] 4.57 \\
\mathrm{Z}[\mathrm{M} \Omega / \mathrm{m}] & 69 . & \mathrm{Rw}[\mathrm{cm}] 3.95 \\
\mathrm{Q} & 19000 . & \mathrm{Rd}[\mathrm{cm}] 3.95 \\
\mathrm{~T} & 0.725 & \mathrm{tw}[\mathrm{cm}] .3 \\
\mathrm{ZT}^{2} / \mathrm{L}[\mathrm{M} \Omega / \mathrm{m}] & 50 . & \mathrm{td}[\mathrm{cm}] 1.65 \\
\mathrm{Z} / \mathrm{Q}[\mathrm{ohm}] & 1500 . & \mathrm{r} \quad[\mathrm{cm}] .7
\end{array}
$$

In figure 2 is reported the plot of the dispersion rclation for the different band-pass showing no overlap between any multipolar mode and the accelerating one.

From that figure is straightforward to see that the accelerating mode is $40 \mathrm{Mhz}$ below the frequency of the nearest dipolar mode.

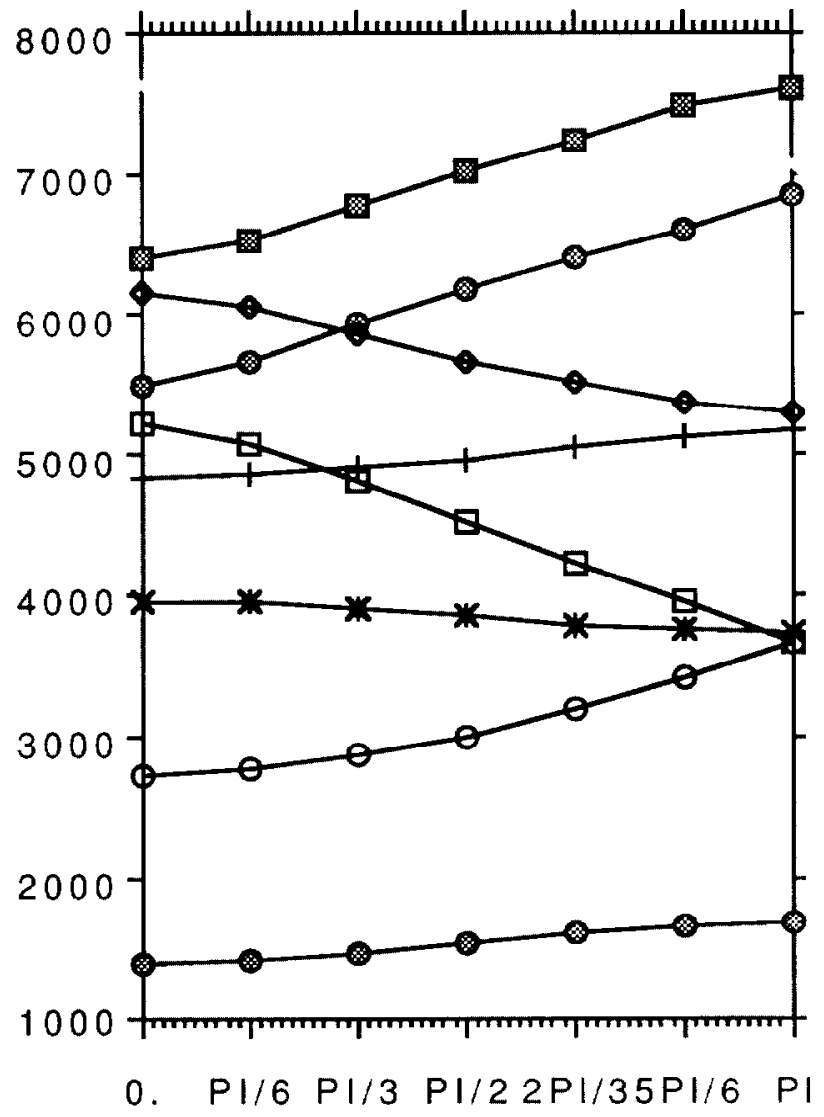

PHASE SHIFT

Figure 2, Dispersion plot for the infinite sructure with superimposed the measured modes of the six cells structure.

\section{EXPERIMENT}

On the basis of our computation we built a small six cell prototype operating at $3.5 \mathrm{GHZ}$ shown in figure 3

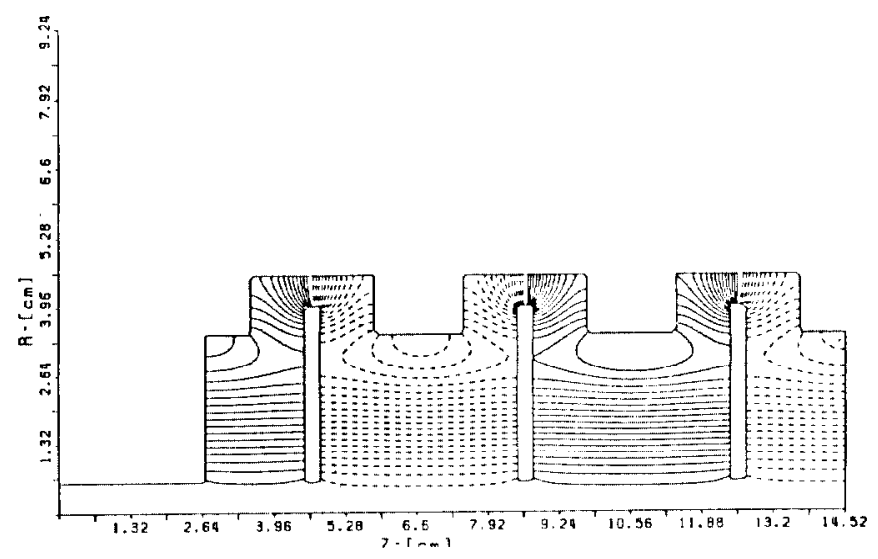

Figure 3, Half six cells ptototype structure.

For that section we measured the resonant frequencies up to eight gigahertz and using a suitable set of 
If probes we identified the bandpass of the dipolar quadrupolar and sextupolar modes.

The measured frequencics and the computed ones for the PI and ZERO modes of the model structure are reported in TABLE II together with the mode band identification.

\section{TABLE II}

\begin{tabular}{|c|c|c|c|}
\hline computed & meausred & error & \multirow{9}{*}{$\begin{array}{l}\text { TM monopol } \\
\text { modes }\end{array}$} \\
\hline Frequency & Frequency & & \\
\hline $\mathrm{MHz}$ & $\mathrm{MHZ}$ & $\%$ & \\
\hline 2743 & 2738 & 0.3 & \\
\hline 3018 & 2995 & 0.7 & \\
\hline 3682 & 3640 & 1.1 & \\
\hline 4517 & 4464 & 1.1 & \\
\hline 5479 & 4438 & 0.7 & \\
\hline 6170 & 3133 & 0.9 & \\
\hline 1645 & 1644 & .06 & \multirow{9}{*}{$\begin{array}{l}\text { Hybrid dipol } \\
\text { modes }\end{array}$} \\
\hline 4011 & 4021 & 0.2 & \\
\hline 4133 & 4134 & .02 & \\
\hline 4956 & 4951 & 0.1 & \\
\hline 5236 & 5252 & 0.3 & \\
\hline 5698 & 5700 & .03 & \\
\hline 6576 & 6500 & 1.1 & \\
\hline 7119 & 7175 & 0.8 & \\
\hline 7759 & 7760 & .01 & \\
\hline 2768 & 2775 & 0.4 & \multirow{8}{*}{$\begin{array}{l}\text { Hybrid quad } \\
\text { modes }\end{array}$} \\
\hline 2880 & 2872 & 0.3 & \\
\hline 5029 & 5048 & 0.4 & \\
\hline 5323 & 5330 & 0.1 & \\
\hline 6135 & 6143 & 0.1 & \\
\hline 6685 & 6670 & 0.2 & \\
\hline 7035 & 7045 & 0.2 & \\
\hline 7962 & 8000 & 0.5 & \\
\hline 4021 & 4041 & 0.5 & \multirow{6}{*}{$\begin{array}{c}\text { Hybrid sext } \\
\text { modes }\end{array}$} \\
\hline 4089 & 4101 & 0.3 & \\
\hline 6163 & 6116 & 0.8 & \\
\hline 6461 & 6490 & 0.5 & \\
\hline 7297 & 7273 & 0.3 & \\
\hline 7945 & 7925 & 0.3 & \\
\hline
\end{tabular}

We also measured for the monopolar modes the axial field distribution and the characteristic impedance $\mathrm{Z} / \mathrm{Q}$ for the accelerating one. the plot of the measured axial field is shown in figure 4 ; the value of the measured characteristic impedance was $\mathrm{Z} / \mathrm{Q}-1500 \mathrm{Ohm}$ in very good agreement the value of 1550 Ohm found by our computations.

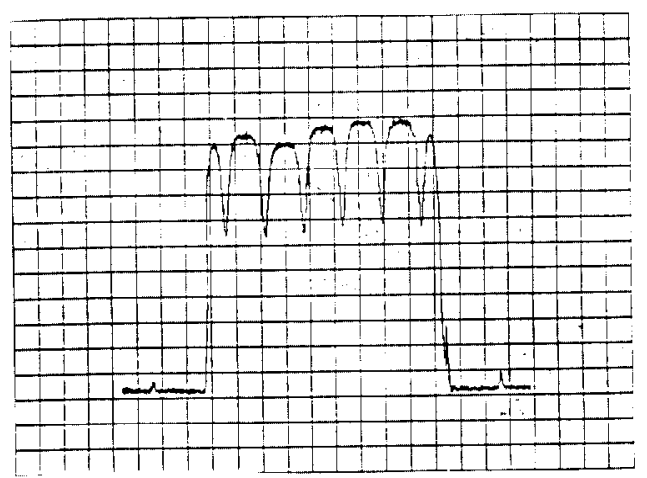

Figure 4 Axial field of the accelereting cavity on the accelerating mode

\section{REFERENCES}

[1] J.J.Manca, E.A.Knapp, D.A.Swenson; "High energy accelerating structures for High gradient proton linac applications"; IEEE Trans. Nucl. Sci., NS-24, 3, June 1977.

[2] S.Inagaki, T.Higo, K.Takata, H.Nakanishi, S.Noguchi, T.Furata, K.Kitagawa, E.Ezura, Y.Kojima, T.Takashima, "Developement of a disk and Washer cavity in KeK ", IEEE Trans. Nuc. Sci., NS-30,(4), 3752(1983).

[3] V.G.Andreev, V.M.Belugin, S.K.Esin, L.V.Kravchuk, V.V.Paramunov, parasitic modes Removal out of operating mode neibourough in the DAWaccelerating Structure" IEEETrans .Nucl. Sci., NS 304) 3575(1986.

4) P.Fernandes, R.Parodi,"Higher order modes computation in RF cavities" IEEE Trans. on Mag mag-24 (1) 154 (1988)

[5] U.van Rienen, T.Weiland, "Triangular discretization method for evaluation of RF fields in cylindrically symmetric cavities". [EEE Trans. Mag. Mag.21.

[6] P.Fernandes, R.Parodi, "On compensation of axial electric field unflatness in multicell accelerating structures", Particle accelerators Vol 14, (1984). 